\title{
Real-life asthma care in Canada
}

\author{
RA Mclvor $\mathrm{MD}^{1}$, J Sampalis $\mathrm{PhD}^{2}$
}

$\mathrm{T}$ he double-blind, randomized, controlled trial (DBRCT) continues to be considered the most valid design for assessment of efficacy. Results of DBRCTs provide the evidence that is used as the basis to approve, or not, a medical intervention for use in the general target population. With a highly selected patient sample that is treated under a rigorous protocol, the DBRCT provides data on the benefits produced by a treatment under ideal conditions. Therefore, issues of adherence and compliance, accessibility to care, presence of comorbid conditions and use of concomitant medications have a minimal impact on the results of DBRCTs.

For several highly prevalent chronic conditions, there is a discrepancy between the efficacy results observed in DBRCTs and the effectiveness observed in a real-life setting under routine clinical practice and patient management. The discrepancy is predominantly in the negative direction, in which the benefits observed in real life are inferior to those anticipated by extrapolating the results of DBRCTs. The main reason for this treatment gap is related to the fact that DBRCTs enroll highly selected patients that are treated and followed up according to a strict regimen, as defined in the study protocol. In addition, medication is provided free of charge and compliance to the required regimen is maximized with protocol-driven manoeuvres. As a result, access to care and compliance have no impact on the study outcomes. Participation of patients in the study is determined by predetermined criteria; thus, the physician's judgment and clinical decision does not affect patient management. Physicians participating in phase II and III DBRCTs are predominantly academics and specialists with strong interest in research and the field.

Conversely, in the real-life setting, access to care and compliance with treatment play a major role in achieving therapeutic goals. The decision regarding which treatment to use and overall management of the patient is made by the treating physician and does not always follow a regimen; nor does it always adhere to treatment guidelines. As a result, there is significant diversity in the type of patients treated with a specific medication. Variability with respect to demographics, comorbidity, concomitant medication use and even socioeconomic status could affect not only compliance with treatment, but also the efficacy of the drug.

The discrepancy between the evidence generated in DBRCTs results observed in the real-life setting presents a significant problem in the assessment of the population based benefit-risk ratio of approved medications. Continued support for the use of the medication requires valid demonstration of real-life benefit and assessment of safety. DBRCTs are typically powered to assess efficacy but do not have sufficient sample size to detect rare, but potentially serious, adverse events. Therefore, there is a need for several welldesigned postmarketing studies that could generate data assessing real-life effectiveness and providing valid evaluation of safety.
Postmarketing studies using either the phase IV or Post Marketing Observational Study (PMOS) design are the essential and the only possible source of data for assessment of real-life effectiveness and safety that could be used to determine population based benefit-risk ratios. Phase IV studies are conducted in the practices of community physicians according to a pre-specified protocol that defines treatment and follow-up procedures. In addition, in phase IV studies, the medication under study is provided by the sponsor at no cost to the patient. As such, the phase IV study may not be considered to be a complete representation of the real-life setting, given that accessibility to care and adherence to treatment guidelines may not be relevant. However, contrary to controlled clinical trials that are conducted by research-oriented clinicians and academics, phase IV studies are conducted by community physicians in their routine clinical practice setting. In addition, while patients enrolled in controlled clinical trials are highly selected to maximize response to treatment and minimize confounding by comorbidity and concomitant medication use, the phase IV patients are those who would be treated with the study medication. Therefore, between-patient variability with respect to medical history profile, predisposing confounders and compliance are more representative of the target population of patients that will receive the medication under investigation in real life. Given these considerations, phase IV studies emulate the real-life setting and their results could be used in the assessment of effectiveness and safety in the real-life setting.

The PMOS, the next step in the assessment of the real-life effectiveness and safety, is also conducted in the practices of community physicians with patients who would be treated with the study medication independently of the study. In the pure PMOS, patients are not treated according to a prespecified protocol but are managed according to the routine practice of the treating physicians. In addition, in the PMOS, the medication is acquired through the regular sources, including health care plans or out of pocket by the patient. Therefore, factors such as accessibility to care, adherence to treatment guidelines and compliance may affect the outcome, and the real-life setting is better represented when compared with the phase IV study.

Based on the above discussion, we can conclude that postmarketing research comprised of phase IV and PMOS closes the loop for drug evaluation and are as important in the overall assessment of benefit-risk as the first phase I and final pivotal phase III studies. Both phase IV and PMOS are necessary, each one providing answers to different questions and incremental value in the assessment of drug therapy. While the PMOS better simulates the reallife study, the phase IV study provides an assessment of effectiveness when accessibility to care is not an issue. Both are necessary

${ }^{1}$ McMaster University, Hamilton, Ontario; ${ }^{2}$ McGill University, Montreal, Quebec

Correspondence: Dr Andrew Mclvor, McMaster University, St Joseph's Healthcare, Firestone Institute for Respiratory Health, T2127, 50 Charlton Avenue East, Hamilton, Ontario L8N 4A6. Telephone 905-522-1155 ext 34330, fax 905-521-6183, e-mail amcivor@stjosham.on.ca 
programs that must be implemented to ensure that marketed drugs provide the benefits expected and that any safety signals are detected early enough to implement corrective actions.

Despite the necessity and utility of postmarketing studies, there remains a publication bias toward DBRCTs, and many high-ranking journals will not consider publishing postmarketing studies regardless of their quality and relevance. This creates a significant information and knowledge gap that does not allow full appreciation of the benefits of approved medications, thus leaving health care providers with limited data upon which to base their clinical decisions. As a result, patients may receive suboptimal care, which in turn increases the burden of illness of the particular disease.

With respect to asthma, treatment guidelines that are based on the evidence generated in clinical trials have been published for the past 20 years. However, their impact on clinical practice has been minimal, with greater than $50 \%$ of the asthma patients remaining uncontrolled and primary care physicians ignoring recommendations for diagnosis and management of asthma $(1,2)$. This is in line with the previous discussion highlighting the difference between the academic participating in DBRCTS and the community general practitioner treating the asthma patients on a regular basis. Access of care and adherence to guidelines also play important roles in this paradigm because the availability of spirometry, the recommended method for diagnosis, is limited because of high cost or difficulty in use.

The Reality of Asthma Control (TRAC) (2) study produced alarming results, showing that very little has changed in the actual real-life management of asthma patients despite the publication of several versions of treatment guidelines and evidence generated in DBRCTs. In this study, the majority (53\%) of the patients with asthma were not controlled, as assessed by their symptoms. Lack of education and appreciation of the importance of an exacerbation by patients and physicians underlie the critical state of asthma management. The latest and largest study to document our failure to improve asthma control outcomes in primary care practice has been the Personal Practice Assessment Program (3). Participants included more than 350 Canadian primary-care settings in which physicians surveyed the asthma control status of more than 10,000 patients (3). Of the surveyed patients, $59 \%$ were considered to be uncontrolled. These uncontrolled patients were six times more likely to have an unscheduled health care visit, almost four times more likely to seek care in an emergency department and twice as likely to be admitted to hospital for out-of-control disease, compared with their controlled counterparts.

Although there are many factors that may explain such suboptimal outcomes, ie, diagnosis and education, treatment for the most art is over-reliant on use of high-dose inhaled corticosteroids (ICS) and early introduction of combination therapy (ICS plus a long-acting bronchodilator in a single inhaler) without checking inhaler technique or adherence. It is easy to forget that use of inhalers is not intuitive; even dry powder inhalers developed to improve delivery (versus meter dose inhalers) need careful instruction and reassessment at each visit.

In recognition of the above treatment gap, which can be, to a large extent, attributed to inadequate translation of evidence to clinical practice, poor adherence to treatment guidelines and lack of exposure of community physicians to training and knowledge transfer for the management of asthma, a position paper known as "The Brussels declaration" (4) calls for real-world studies to assess regional variations in practice patterns for the management of asthma, with emphasis on knowledge dissemination and patient education, as well as increased involvement in their care.

The Singulair Add-on Study (SAS) (5), SIngulair in Mild asthma: comPLiance and Effectiveness (SIMPLE) (6) and SingulaiR in Asthma anD Allergic Rhinitis (RADAR) (7) studies, in this supplement to the Canadian Respiratory Journal, address this need for well-designed postmarketing phase IV studies assessing the real-life effectiveness of asthma management regimens. The SAS trial demonstrated the real-life effectiveness of montelukast as an add-on therapy in patients that were uncontrolled or not satisfied with ICS monotherapy. In this study, the improvement of compliance with treatment was noted and this could at least in part account for the improved control of the asthma symptoms. In the SIMPLE trial, patients that were not controlled on ICS monotherapy were switched to montelukast monotherapy. Control of asthma symptoms as well as compliance with treatment improved significantly after initiation of treatment with montelukast. The RADAR study demonstrated that montelukast as an add-on to ICS or LABA was effective in controlling asthma in asthma patients with allergic rhinitis.

These studies will hopefully add front line information on approaches to asthma management outside specialist practice, and supplement and balance crucial information from efficacy clinical trials. Taken collectively, these studies provide evidence of the real-life effectiveness of montelukast in the management of asthma patients. The real-life setting allows physicians to make inference from these studies to their patient populations. The results also have important implications from a public health perspective as well as for health care stakeholders and decision makers.

\section{REFERENCES}

1. McIvor RA, Chapman RK. The coming of age of asthma guidelines. Lancet 2008;372:1021-2.

2. McIvor RA, Boulet L-P, FitzGerald MJ, Zimmerman S, Chapman KR. Asthma control in Canada. Can Fam Physician 2007;53:672-7.

3. Chapman KR, Boulet LP, Rea RM, Franssen E. Suboptimal asthma control: Prevalence, detection and consequences in general practice. Eur Resp J 2008;31:320-5.

4. Holgate S, Bisgard H, Bjermer L, et al. The Brussels declaration: The need for change in asthma management. Eur Respir J 2008;32:1433-42.

5. FitzGerald JM, Foucart S, Coyle S, et al. Montelukast as add-on therapy to inhaled corticosteroids in the management of asthma (the SAS trial). Can Respir J 2009;16(Suppl A):5A-10A.

6. McIvor RA, Kaplan A, Koch C, Sampalis JS. Montelukast as an alternative to low-dose inhaled corticosteroids in the management of mild asthma (the SIMPLE trial): An open-label effectiveness trial. Can Respir J 2009;16(Suppl A):11A-16A.

7. Keith PK, Koch C, Djandji M, et al. Montelukast as add-on therapy with inhaled corticosteroids or inhaled corticosteroids and longacting beta-2-agonist treatment in the management of patients diagnosed with asthma and concurrent allergic rhinitis (the RADAR trial). Can Respir J 2009;16(Suppl A):17A-24A. 


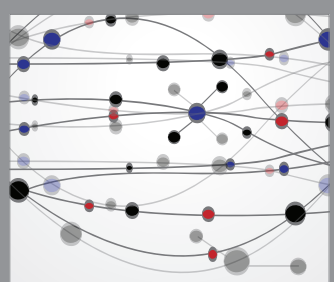

The Scientific World Journal
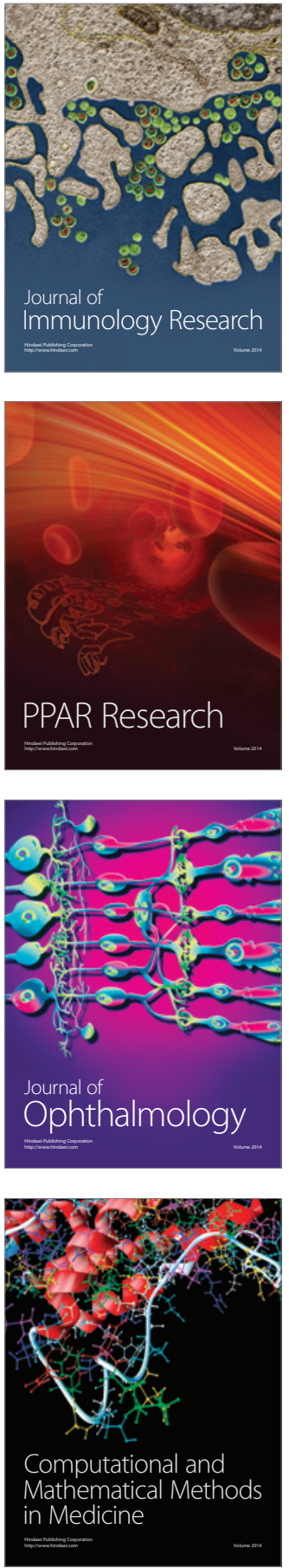

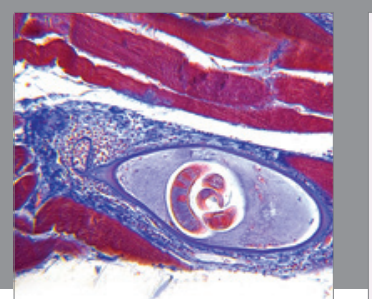

Gastroenterology Research and Practice

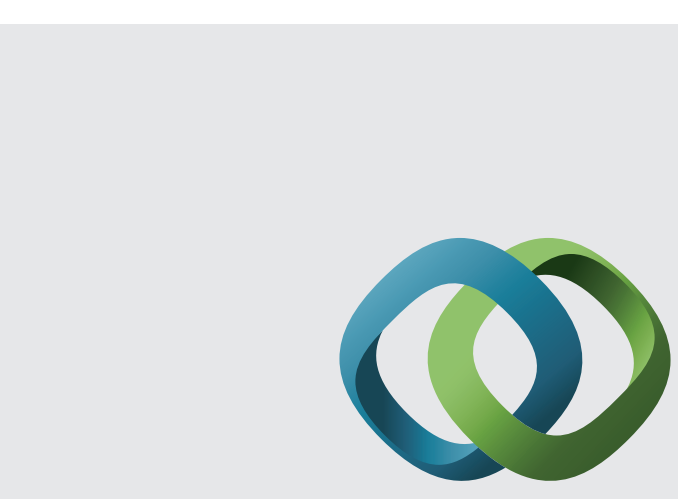

\section{Hindawi}

Submit your manuscripts at

http://www.hindawi.com
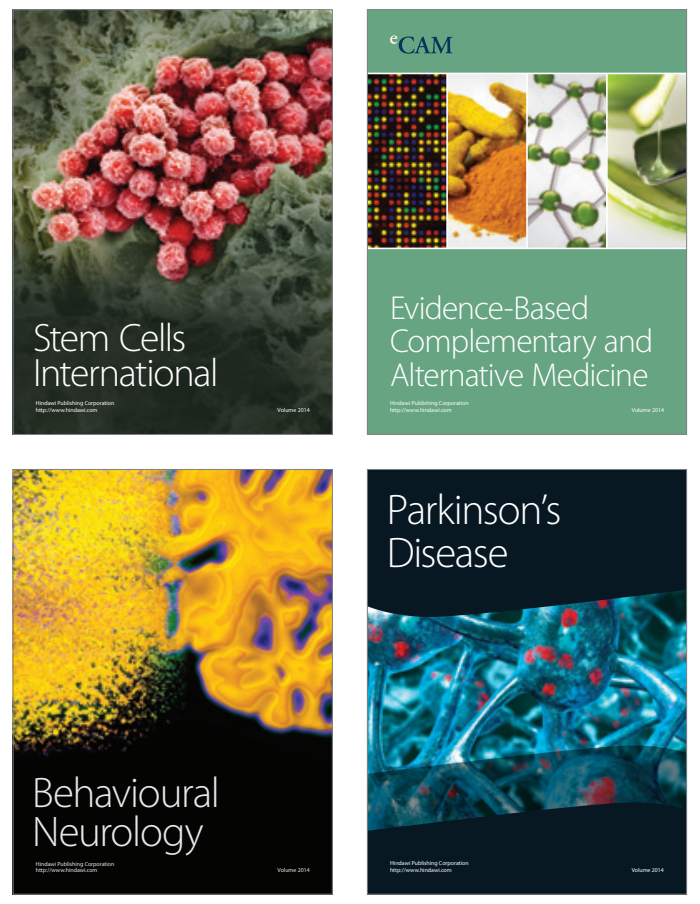
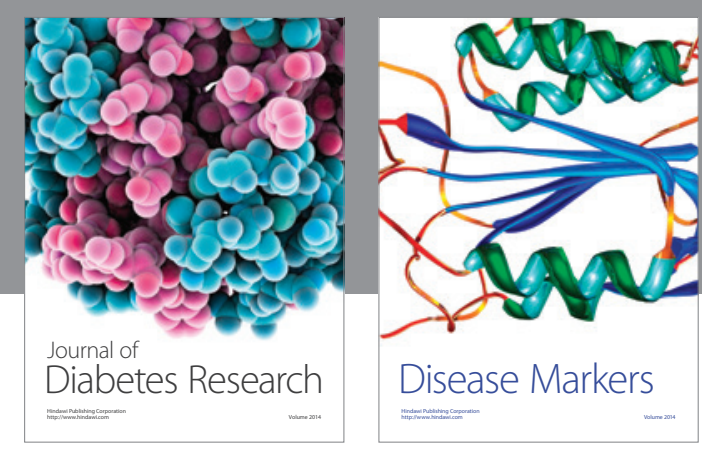

Disease Markers
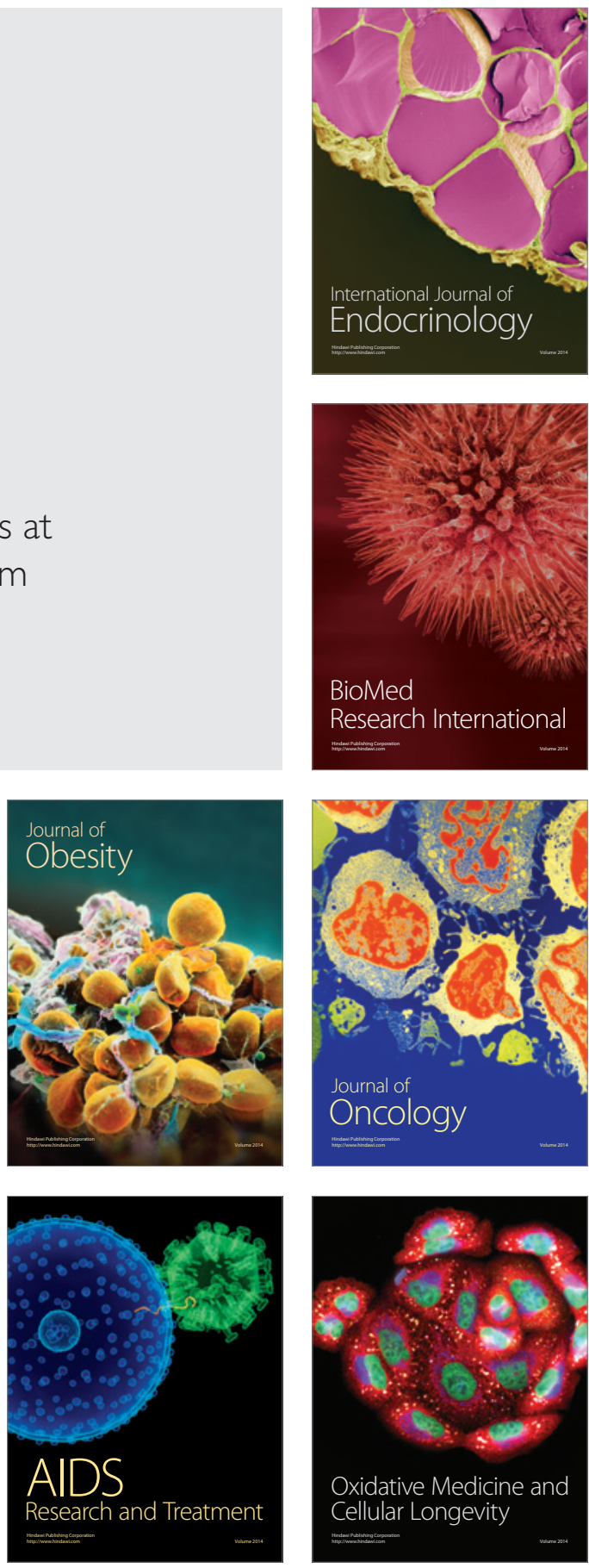